\title{
Publisher Correction: Impacts of hydrothermal plume processes on oceanic metal cycles and transport
}

Amy Gartman (1) and Alyssa J. Findlay (1)

Correction to: Nature Geoscience https://doi.org/10.1038/s41561-020-0579-0, published online 1 June 2020.

In the version of this Perspective originally published, the last two sentences of Box 1 were incorrectly edited. The following sentences:

'The size of the pie charts represents the calculated flux at the point of emission and there is relatively good agreement between estimates for $\mathrm{Fe}$ and $\mathrm{Mn}$, despite loss during plume transport. However, for $\mathrm{Zn}$ a reconciliation between these datasets requires further work as the effective flux is in excess of the initial emission and, while the $\mathrm{Cu}$ flux reflects relative loss from within the plume, the calculated total flux based on far-field data exceeds estimates based on near-vent data ${ }^{14,51,60-63}$. should have been:

'The size of the pie charts is proportional to the flux, at a log scale; the wedge represents the percent of initial flux remaining in the far field. $\mathrm{Fe}, \mathrm{Cu}$ and $\mathrm{Mn}$ demonstrate loss during plume transport. However, for $\mathrm{Zn}$ a reconciliation between these datasets requires further work as the effective flux is in excess of the initial emission ${ }^{14,51,60-63}$.

These have now been corrected.

Published online: 21 July 2020

https://doi.org/10.1038/s41561-020-0625-y

๑) Springer Nature Limited 2020 